05

\title{
Магнитное торможение и энергетические потери в бесконтактных подшипниках на основе сверхпроводящих лент
}

\author{
() А.И. Подливаев, И.А. Руднев \\ Национальный исследовательский ядерный университет МИФИ, \\ 115409 Москва, Россия \\ e-mail: iarudnev@mephi.ru
}

Поступило в Редакцию 2 июля 2018 г.

В окончательной редакции 2 июля 2018 г.

Принято к публикации 28 октября 2019 г.

Рассмотрены проблемы магнитного торможения и возникновения энергетических потерь в бесконтактных подшипниках на основе высокотемпературных сверхпроводящих лент. Рассмотрены модельные конфигурации подшипников, в которых сверхпроводящая лента является статором, а ротор представляет собой набор постоянных магнитов. Показано, что магнитным трением можно пренебречь в случае, когда число постоянных магнитов в роторе более восьми. Данный результат указывает на возможность создания масштабированных магнитных подшипников для систем долговременного хранения энергии, например, кинетических накопителей.

Ключевые слова: высокотемпературные сверхпроводящие ленты, магнитные подшипники, трение.

DOI: $10.21883 / J T F .2020 .04 .49082 .261-18$

\section{Введение}

Применение в бесконтактных подшипниках пары сверхпроводник/постоянный магнит перспективно не только по причине отсутствия в таких подшипниках сил трения. Отсутствие прямого механического контакта между движущимися частями позволяет эффективно использовать магнитные подшипники в высокооборотной технике: кинетических накопителях, гироскопах и аналогичных устройствах (см., например, [1]).

Изготовление сверхпроводящего ротора (статора) из объемного высокотемпературного сверхпроводника (ВТСП) является достаточно сложной технологической задачей. Во-первых, ВТСП материал достаточно хрупкий (в данном случае это перовскит ҮВСО). Во-вторых, требуется сложный режим отжига, обеспечивающий однородность сверхпроводящих свойств материала. Альтернативой объемному сверхпроводнику может стать наборный сверхпроводник, состоящий из стопок ВТСП лент [2]. Аналогом стопки лент можно считать также короткозамкнутые катушки, изготовленные из ВТСП лент [3], которые могут применяться в различных левитационных приложениях. Использование ВТСП лент вместо объемных материалов имеет ряд преимуществ. Технология создания сверхпроводящего композита произвольной формы, набранного из лент, более проста. Сверхпроводящие характеристики стопки лент не уступают объемному материалу [4]. Кроме того, ВТСП ленты обладают более высокими по сравнению с объемными материалами механическими свойствами.

Изготовление магнитного ротора также представляет технологическую проблему. Цельный магнит желаемой формы и размера должен обеспечивать магнитное поле аксиальной симметрии. Альтернативой цельному магни- ту (как и в случае со сверхпроводником) может стать магнит наборный. Под наборным магнитом подразумевается мозаика, состоящая из большого числа небольших магнитов. Каждый элемент мозаики представляет собой однородно намагниченный ферромагнетик, имеющий форму прямоугольного параллелепипеда. Недостатком такой мозаики является неоднородность магнитного поля, особо заметная на стыках соседних элементов. Неоднородность магнитного поля может вызвать гистерезисное перемагничивание сверхпроводника. Перемагничивание сверхпроводника сопровождается рассеянием энергии (см., например, обзор [5]) и, следовательно, возникновением силы трения в подшипнике. Предметом настоящей работы является теоретическое исследование влияния неоднородности магнитного поля наборного магнита на силу трения в подшипнике, в котором в качестве сверхпроводящего статора будут рассмотрены однослойные и многослойные кольца, сформированные из ВТСП лент второго поколения.

\section{Описание модели подшипника. Геометрия. Основные уравнения}

Рассматриваемый нами подшипник состоит из ротора и статора. Деление на „ротор“ и „статор“ условное. Возможно, как вращение внутреннего вала подшипника при неподвижной внешней части, так и вращение внешней части вокруг неподвижной внутренней оси. Для определенности в дальнейшем полагаем неподвижным статором сверхпроводящую часть подшипника, а ротор формируется из набора постоянных магнитов малого размера. Этот выбор обусловлен, во-первых, тем, что охлаждать технически проще неподвижный 


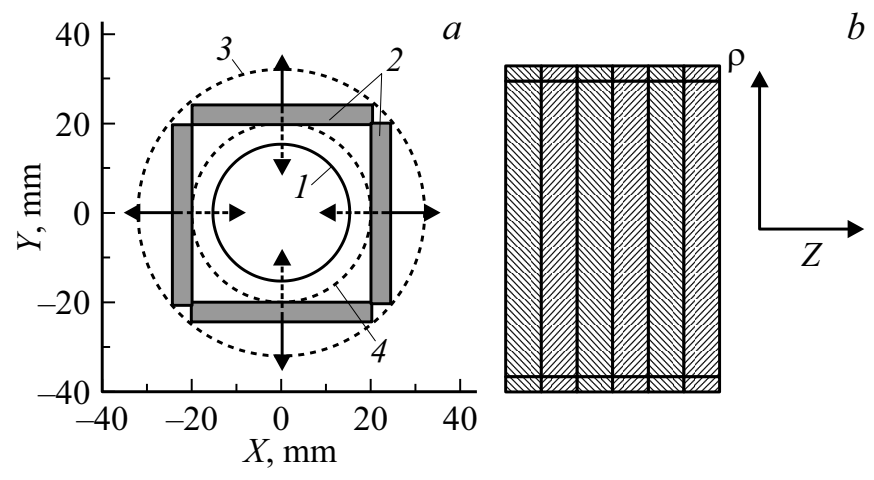

Рис. 1. Подшипник с четырехугольным магнитным ротором. $a-$ вид с торца: $1-$ сверхпроводящий статор, $2-$ магнитный ротор, 3 и $4-$ внешняя и внутренняя границы магнитного ротора (при его вращении) соответственно. Сплошными и штриховыми стрелками на магнитах указано направление намагниченности для соседних (в направлении оси $Z$ ) магнитов. $b-$ магнитный ротор (6 магнитных колец в направлении оси $Z$ ). Вид сбоку. Различной штриховкой отмечены магниты с магнитным моментом внутрь и наружу ротора соответственно.

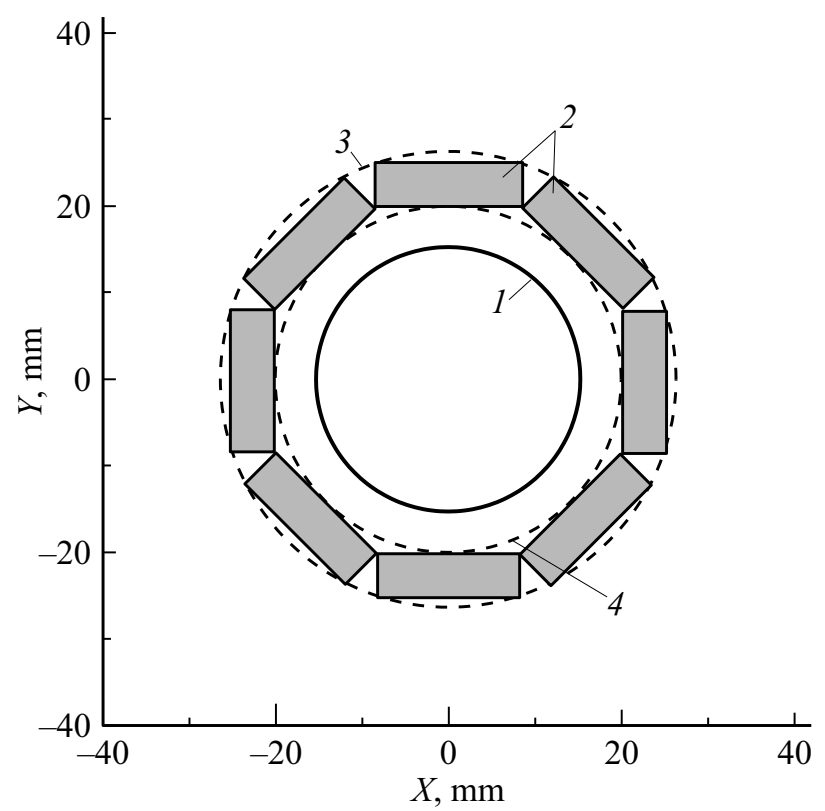

Pис. 2. Подшипники с восьмиугольным магнитным ротором. Вид с торца: $1-$ сверхпроводящий статор, $2-$ магнитный ротор, 3 и 4 - внешняя и внутренняя границы магнитного ротора (при его вращении) соответственно.

сверхпроводник. Возможность охлаждения позволяет пренебречь перегревом сверхпроводника (в противном случае возможно возникновение термодинамической нестабильности [6,7]). Во-вторых, вращающийся массивный магнитный ротор вокруг внутренней сверхпроводящей оси оптимален при создании гироскопа. На рис. 1,2 представлены подшипники с четырехугольными и восьмиугольными роторами соответственно. В дальнейшем число магнитов в роторе обозначает- ся как $N_{\text {mag }}\left(N_{m a g}=4,8,16\right)$. Для расчетов выбраны следующие размеры магнитного ротора. Внутренняя граница ротора (внутренняя окружность, обозначенная штриховой линией на рис. 1 и 2) имеет радиус $20 \mathrm{~mm}$ как для четырехугольного, так и для восьмиугольного и шестнадцатиугольного роторов. Внешняя граница ротора (внешняя окружность, обозначенная штриховой линией на рис. 1 и 2) имеет радиусы 32.016 и $26.34 \mathrm{~mm}$ для четырехугольного и восьмиугольного роторов соответственно. Одинаковыми для всех роторов являются также толщина магнитов, равная $5 \mathrm{~mm}$ (рис. $1, a, 2$ ), а также ширина магнитов $d_{Z}$, равная $12 \mathrm{~mm}$ в направлении оси $Z$ (рис. 1,b). При выборе параметров ленты мы ориентировались на достаточно перспективную в технических приложениях (например, в ограничителях тока [8]) 12-ти миллиметровую ленту СуперОкс. Намагниченность каждого магнита считаем однородной. Если вектор плотности магнитного момента в точке пространства с радиус-вектором $\mathbf{r}^{\prime}$ обозначить $\mathbf{m}\left(\mathbf{r}^{\prime}\right)$, вектор индукции магнитного поля в точке $\mathbf{r}$, создаваемого магнитом (который занимает объем $V$ ), имеет следующий вид [9]:

$$
\begin{gathered}
\mathbf{B}(\mathbf{r})=\mu_{0} \int_{V}\left[\frac{3 \mathbf{n}\left(\mathbf{n}, \mathbf{m}\left(\mathbf{r}^{\prime}\right)\right)-\mathbf{m}\left(\mathbf{r}^{\prime}\right)}{\left|\mathbf{r}-\mathbf{r}^{\prime}\right|^{3}}\right] d^{2} r^{\prime}, \\
\mathbf{n}=\left(\mathbf{r}-\mathbf{r}^{\prime}\right) /\left|\mathbf{r}-\mathbf{r}^{\prime}\right| .
\end{gathered}
$$

Модуль вектора $\mathbf{m}\left(\mathbf{r}^{\prime}\right)$ подбирался так, чтобы величина индукции магнитного поля отдельного магнита (рис. 1) в центре его поверхности была равна $0.3 \mathrm{~T}$.

Обычная толщина ВТСП ленты $\sim 0.1 \mathrm{~mm}$, поэтому в настоящей работе так же как в работе [4] мы считаем толщину обмотки статора нулевой. Принимаем, что плотность критического тока в однослойном сплошном ВТСП кольце равна $j_{c}$ в двухслойном $-2 j_{c}$. Плотность критического тока на границах соседних колец, перпендикулярных оси $Z$, равна нулю. Координаты $Z$ границ колец статора совпадают с границами разнополярных магнитов ротора. Эти границы имеют координаты $Z=0$, $\pm d_{Z} / 2, \pm 2 d_{Z} / 2, \pm 3 d_{Z} / 2, \pm 4 d_{Z} / 2$, и т.д.

При большом количестве магнитов в роторе $\left(N_{\text {mag }} \gg 1\right)$ и малой шириной ВТСП колец по сравнению с их периметром $\left(d_{p} / d_{Z} \gg 1\right)$ кривизной статора можно пренебречь и заменить задачу определения плотности сверхпроводящего тока, поставленную в цилиндрических координатах $(\rho, \phi, Z)$ при $\rho=R_{S}$, двумерной задачей в плоскости $(p, Z), p=R_{S} \phi$. Областью определения магнитного поля и плотности токов в этом случае является прямоугольник $-d_{Z} / 2 \leq Z \leq d_{Z} / 2$, $-d_{p} / 2 \leq p \leq d_{p} /$. Граничные условия - периодические как по угловой переменной $p$, так и по переменной $Z$ (полагается, что ротор состоит из бесконечного числа прилежащих друг к другу колец разнополярных магнитов). Замена цилиндрической задачи на плоскую обоснована тем, что, во-первых, отношение $d_{p} / d_{Z}=8$ достаточно велико, во-вторых, с практической стороны 


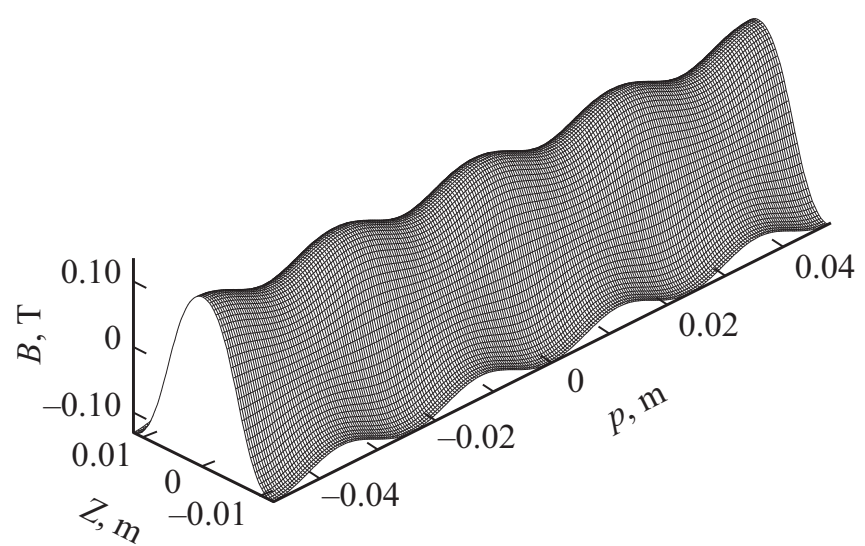

Рис. 3. Нормальная компонента индукции магнитного поля $B_{\rho}$ на развернутой поверхности статора.

наибольший интерес представляют роторы с большим количеством магнитов $\left(N_{\text {mag }}=8\right.$ и 16$)$. Четырехугольный ротор мы рассматриваем для качественного определения зависимости силы трения подшипника от числа магнитов. К тому же именно для четырехугольного ротора наиболее ярко проявляется влияние неоднородности внешнего поля (по угловой переменной) на распределение сверхпроводящих токов.

Внешнее магнитное поле магнитного ротора в статоре рассчитывалось по формуле (1). Нормальная к поверхности статора компонента индукции магнитного поля на этой поверхности представлена на рис. 3. Индукция определялась для четырехугольного ротора.

Вариация амплитуды магнитного поля $\left(B_{\max }-B_{\min }\right) /\left(B_{\max }+B_{\min }\right) \quad$ при координате $Z=0$ равна $14.8,1.3$, и $0.2 \%$ для $N_{\text {mag }}=4,8$ и 16 соответственно. Идея применения неоднородного (периодического) внешнего магнитного поля не нова. Так, в работах $[10,11]$ для создания периодической конфигурации использовался набор магнитов разной полярности, однако особенностью настоящей работы является расчет ВТСП ленты в слабо изменяющемся поле, которое, однако, воздействует на сверхпроводник в течение неограниченного числа периодов.

Полевую зависимость критического тока сверхпроводящей ленты мы описываем в рамках двухэкспоненциальной модели [12], в которой плотность поверхностных токов $j_{c}(B)$ ВТСП пленки имеет следующий вид:

$$
j_{c}(B)=A_{1} \exp \left(-|B| / \beta_{1}\right)+A_{2} \exp \left(-|B| / \beta_{2}\right)
$$

с параметрами $A_{1}=12.9 \mathrm{kA} / \mathrm{m}, A_{2}=13.8 \mathrm{kA} / \mathrm{m}$, $\beta_{1}=0.08 \mathrm{~T}, \beta_{2}=1.92 \mathrm{~T}$. Шаг пространственной сетки в представленных расчетах $\delta h \sim 0.375 \mathrm{~mm}$. Расчет проводился в рамках модели критического состояния сверхпроводника, исходно предложенной в работе [13], некоторые модификации и приложения которой даны в обзоре [4]. Подробное описание постановки математической задачи и алгоритма численного счета для полевой зависимости (2) даны в публикациях $[4,14]$, и в настоящей работе не приводятся вследствие громоздкости.

Режим изменения внешнего магнитного поля был следующий. На первом этапе магнитное поле, пропорциональное полю ротора без вращения ротора, монотонно увеличивалось от нулевого значения до значения, определяемого выражением (1). Далее проводилось вращение ротора на 40 оборотов (количество оборотов далее обозначается как $\left.N_{\text {rot }}\right)$. При этом определялась соответствующая плотность сверхпроводящих токов. Один оборот цилиндрической конфигурации соответствует сдвигу магнитного поля в плоской задаче по переменной $p$ на величину периода $d_{p}$. При этом сдвиге тангенциальная компонента силы взаимодействия поля ротора $B_{\rho}$ с токами статора (сила трения) $f_{p}$ для одного кольца статора определялась из закона Ампера

$$
f_{p}=\int_{-d_{p} / 2}^{d_{p} / 2} d p \int_{-d_{Z} / 2}^{d_{Z} / 2} B_{\rho}(p, Z) j_{Z}(p, Z) d Z .
$$

\section{Результаты}

Зависимость силы трения от числа оборотов ротора для четырехугольного статора $\left(N_{m a g}=4\right)$ представлена на рис. 4. На рисунке видно, что сила трения устанавливается за один оборот. Для роторов с большим числом магнитов $\left(N_{m a g}=8\right.$ и 16$)$ установление также происходит менее чем за два оборота. В настоящей работе нас не интересует характер переходных процессов. Поэтому определение силы трения мы производим усреднением этой величины с третьего по десятый оборот. В случае четырехугольного ротора $\left(N_{\text {mag }}=4\right)$ сила трения для однослойного/двуслойного

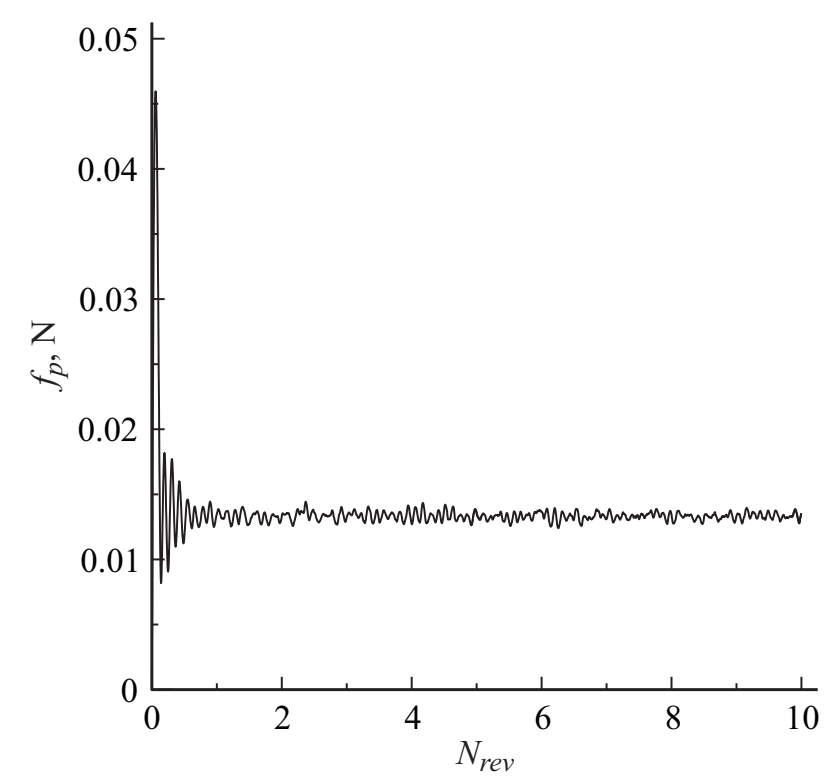

Рис. 4. Зависимость силы трения от числа оборотов четырехугольного ротора. 


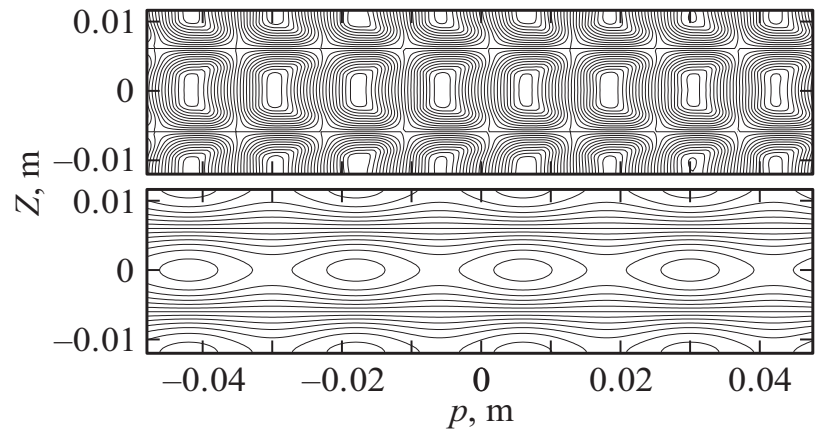

Рис. 5. Линии сверхпроводящих токов (верхняя часть рисунка) и линии уровней нормальной к поверхности статора компоненты магнитного поля четырехугольного ротора (нижняя часть рисунка). Одна линия тока на верхнем рисунке соответствует току $5 \mathrm{~A}$. Одна линия тока на нижнем рисунке соответствует индукции магнитного поля $0.02 \mathrm{~T}$.

статора равна 0.013 и $0.0021 \mathrm{~N}$ соответственно. В случае восьмиугольного ротора $\left(N_{\text {mag }}=8\right)$ сила трения для однослойного/двуслойного статора равна $2.01 \cdot 10^{-6}$ и $1.25 \cdot 10^{-6} \mathrm{~N}$ соответственно. При $N_{\text {mag }}=16$ сила трения для однослойного/двуслойного статора равна $1.5 \cdot 10^{-7}$ и $8.1 \cdot 10^{-8} \mathrm{~N}$ соответственно.

Характерное распределение плотности сверхпроводящих токов в статоре и магнитного поля четырехугольного ротора представлены на рис. 5. Верхняя часть рисунка представляет линии сверхпроводящих токов, нижняя - линии уровней магнитного поля ротора. На этом рисунке представлен фрагмент статора шириной $2 d_{Z}$ (центральная лента окружена сверху и снизу половинами соседних лент статора). При повороте ротора происходит периодическое смещение картины по оси $p$.

Для качественной интерпретации зависимости силы трения от числа магнитов ротора и витков ВТСП ленты на статоре удобно представить статор системой взаимосвязанных магнитных насосов. Представим каждую ячейку счетной сетки размером $h \times h$ на статоре как элементарный магнитный насос. Если обозначить частоту вращения ротора символом $\omega$, частота накачки каждого насоса внешним переменным магнитным полем будет равна $\omega N_{\text {mag }}$ (фаза колебаний поля для ячеек с различной координатой $p$ будет различной). Наведенный внешним полем магнитным момент ячейки равен $g \mathbf{n}_{\rho} h^{2}$. В этом выражении $\mathbf{n}_{\rho}-$ единичный вектор нормали к поверхности статора. Величина $g$ - плотность магнитного момента, связанная с плотностью поверхностных токов статора $\mathbf{j}(p, Z)$ соотношением $\mathbf{j}(p, Z)=\nabla\left(g \mathbf{n}_{\rho}\right)$ (см. обзор [5] и литературу к нему). На рис. 6 представлена зависимость плотности магнитного момента ячейки с координатами $p=0, Z=0$ от величины магнитной индукции ротора в этой точке. Верхняя кривая соответствует однослойному, а нижняя - двуслойному ВТСП статору. Зависимости приведены для одного оборота ротора после прохождения переходных процессов.
Величина индукции магнитного поля в данной точке изменяется от минимального $B_{\min }=0.095$ Т до максимального $B_{\max }=0.128 \mathrm{~T}$ значения.

На участке кривой, соответствующей возрастанию индукции поля от минимального $B_{\min }$ до максимального $B_{\max }$ значения, ЭДС изменяющегося поля наводит токи, уменьшающие плотность магнитного момента (наведенное магнитное поле противоположно полю наводящему). Вследствие этого магнитный момент минимален при максимальном магнитном поле. Процесс экранирования ограничен конечным значением критического тока (в противном случае экранирование было бы полным во всем статоре). При уменьшении магнитного поля от максимального значения на начальном этапе ЭДС изменяет знак, и плотность тока в данной точке становится меньше критической. При дальнейшем уменьшении поля плотность тока проходит через нуль, снова ее величина достигает критического значения. Амплитуда изменения индукции магнитного поля четырехугольного ротора на поверхности статора достигает $14.8 \%$ от среднего значения. Этого изменения достаточно для того чтобы, во-первых, в однослойном статоре магнитный момент элементарной ячейки изменял знак (рис. 6 , верхняя кривая) при том, что магнитное поле знакопостоянно. Вовторых, малость критического тока при таком изменении поля имеет следствием радикальное перемагничивание статора, что приводит к широкой гистерезисной петле (как следствие, к диссипации энергии и большой силе трения). Двукратное увеличение плотности критического тока в двуслойном статоре блокирует процесс перемагничивания (рис. 6, нижняя кривая). Площадь ги-

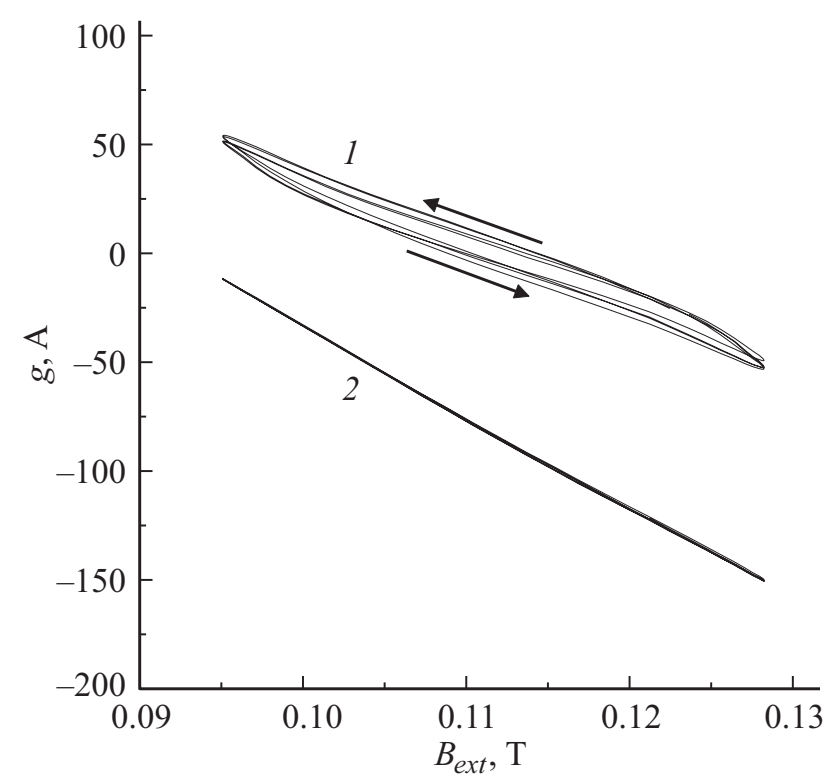

Рис. 6. Зависимость плотности магнитного момента в точке с координатами $p=0, Z=0$ от величины индукции магнитного поля для одного оборота ротора. 1 - зависимость для однослойного статора, 2 - зависимость для двухслойного статора. Стрелками указано направление обхода гистерезисной кривой при вращении ротора. 
стерезисной петли резко уменьшается, и, как следствие, уменьшается сила трения. Аналогично объясняется существенное уменьшение силы трения при увеличении числа магнитов. Вариации амплитуды магнитного поля в 1.3 и $0.2 \%$ для $N_{m a g}=8$ и 16 уже недостаточно для перемагничивания даже однослойного статора.

\section{Выводы}

Проведенные расчеты показали, что сила магнитного трения и возникающие при этом энергетические потери не являются препятствием в создании комбинированного подшипника, ротор которого состоит более чем из 8 магнитов, а статор - из нескольких короткозамкнутых слоев ВТСП ленты второго поколения. Экспериментальная проверка сделанного вывода, а также анализ влияния иных параметров подшипника (эксцентричное расположение и перекос оси ротора относительно оси статора, дефекты ВТСП ленты статора и т.п.), которые могут критически ухудшить его характеристики, будут проведены отдельно.

Следует отметить, что рассмотренная конфигурация магнитного ротора и сверхпроводящего статора из ВТСП ленты имеет принципиальное преимущество по сравнению с подшипниками на объемных ВТСП, так как позволяет проводить практически неограниченное масштабирование устройства.

\section{Финансирование работы}

Исследование выполнено за счет гранта Российского научного фонда (проект № 17-19-01527).

\section{Конфликт интересов}

Авторы заявляют, что у них нет конфликта интересов.

\section{Список литературы}

[1] Miyazaki Y., Mizuno K., Yamashita T., Ogata M., Hasegawa H., Nagashima K., Mukoyama S., Matsuoka T., Nakao K., Horiuch S., Maeda T., Shimizu H. // Cryogenics. 2016. Vol. 80. P. 234-237.

[2] Kun Liu, Wenjiao Yang, Guangtong Ma, Lö̈c Quéval, Tianyong Gong, Changqing Ye, Xiang Li, Zhen Luo // Supercond. Sci. Technol. 2018. Vol. 31. P. 015013.

[3] Козинщева М.В., Бишаев А.М., Буш А.А., Гавриков М.Б., Каменцев К.Е., Нижельский Н.А., Савельев В.В., Сигов А.С. // ЖТФ. 2017. Т. 87. Вып. 6. С. 875.

[4] Rudnev I.A., Podlivaev A.I. // IEEE Transactions on Appl. Superconduct. 2016. Vol. 26. N 4. 8200104.

[5] Jooss Ch., Albrecht J., Kuhn H., Leonhardt S., Kronmuller H. // Rep. Prog. Phys. 2002. Vol. 65. P. 651.

[6] Романовский В.P. // ЖТФ. 2017. Т. 87. Вып. 1. С. 49.

[7] Романовский В.Р. // ЖТФ. 2017. Т. 87. Вып. 4. С. 540.

[8] Алферов Д.Ф., Ахметгареев М.Р., Евсин Д.В., Волошин И.Ф., Калинов А.В., Фишер Л.М., Цхай Е.В. // ЖТФ. 2018. Т. 88. Вып. 1. С. 28.
[9] Ландау Л.Д., Лифиии Е.M. // Теория поля. М.: Наука, 1988. $512 \mathrm{c}$.

[10] Базаров Б.А., Ежсов В.Ф., Коврижных Н.А., Рябов В.Л., Андреев А.З., Глушков А.Г., Князьков В.А., Крыгин Г.Б. // Письма в ЖТФ. 2016. Т. 42. Вып. 13. С. 1.

[11] Подливаев А.И., Покровский С.В., Анищенко И.В., Руднев И.А. // Письма в ЖТФ. 2017. Т. 43. Вып. 24. С. 96.

[12] Подливаев А.И., Руднев И.А., Шабанова Н.П. // Краткие сообщения по физике ФИАН. 2014. Т. 41. Вып. 12. С. 13.

[13] Bean C.P. // Phys. Rev. Lett. 1962. Vol. 8. P. 250.

[14] Podlivaev A., Rudnev I. // Supercond. Sci. Technol. 2017. Vol. 30. P. 035021. 